\title{
Getting from here to there
}

\section{Essays in Biochemistry}

Molecular Trafficking

Essays in Biochemistry: Molecular Trafficking

edited by P. Bernstein

Portland Press · September 2000

Paperback $£ 19 / \$ 24$

Lucy Erin O'Brien and Keith Mostov

G etting proteins to the right place at the right time is an immense logistical challenge for cells. Each protein must be guided to its proper site, and this site can change over the lifetime of the protein. For example, a secreted protein might acquire post-translational modification in the endoplasmic reticulum (ER) followed by a layover in the Golgi before reaching the cell surface. A chloroplast protein might translocate across as many as three lipid bilayers to reach its final destination. A transcription factor might shuttle into and out of the nucleus in response to signals to alter gene expression. The scope of this transport problem is vast; the genome project estimates that $30 \%$ of all proteins travel across membranes. It is likely that some aspect of protein trafficking touches on the research of every cell biologist.

The past thirty years have revolutionized our understanding of the molecular mechanisms that cells use to orchestrate complex intracellular itineraries. We have identified key players such as signal recognition particle, coat proteins and SNARES, and elucidated much about their roles in protein targeting. This work is now part of the cell biology canon, described in venerated textbooks such as Molecular Cell Biology by H. Lodish et al. (W. H. Freeman, 1999) or Molecular Biology of the Cell by B. Alberts et al. (Garland, 1994). Although appropriate for an introductory survey course in cell biology, these texts of necessity lack the depth of analysis required for an advanced undergraduate or introductory graduate course in protein kinesis. Essays in Biochemistry: Molecular Trafficking seeks to fill this niche. The book's ten chapters, each by a different author, discuss chosen aspects of macromolecular movement at a level between the standard textbooks and the typical journal review article.

For the most part, this book succeeds admirably in its intent. The writing is lucid and offers a contemporary view that balances dogma and speculation. Chapters by Suzanna Meacock et al. on protein translocation into the ER, and by Eric Schwoebel and Mary Shannon Moore on the control of gene expression by regulated nuclear transport, provide timely discussions of these fast-moving areas. Other chapters, such as those by Peter Scheiffele and Joachim Füllekrug on glycosylation and protein transport, and by Matthew E. Harris and Thomas J. Hope on RNA export, seamlessly incorporate experimental history into their narratives, providing an invaluable perspective on the evolution of current thought in their fields. Overall, advanced students will find the volume's sophisticated yet accessible approach makes it an ideal companion to primary research papers. Investigators newly drawn into the field will also benefit and will value the self-contained nature of the various chapters.

However, Molecular Trafficking does have its shortcomings. Its major fault stems from this division into stand-alone chapters that are written by separate authors. Although Philip Bernstein's preface touches on some general themes, the volume sorely lacks a meatier overview that compares how different translocation systems solve common problems such as signal recognition, energy usage, and mono- or bi-directionality. A chapter on prokaryotic translocation systems is markedly absent and would have underscored the evolutionary unity of translocation systems across the biological spectrum. Additionally, the information included in four short chapters on nuclear-cytoplasmic transport is redundant; condensation to one or two longer chapters would have been more effective. While two-colour schematic illustrations are clear and abundant, the book regrettably omits electron micrographs and crystal structures despite the tremendous advancements that electron microscopy and crystallography have bestowed on the field. A high-resolution image of the nuclear pore complex, for instance, would have been informative.

Although not its primary motive, Molecular Trafficking also offers the astute reader a lesson in history: namely, the inescapable influence of Günter Blobel on the field of protein trafficking. The 1999 Nobelist's impact resonates in nearly every chapter, from his classic studies on ER translocation and the nuclear pore to his lesser-known but seminal findings on chloroplast and mitochondrial import. Not surprisingly, many of the authors are the scientific children or grandchildren of Blobel. Thus, the volume is not only a guide for those who pursue an understanding of molecular trafficking, but also a testament to the greatness of Blobel's legacy.

Lucy Erin O'Brien and Keith Mostov are at the University of California, San Francisco, California 94143, USA

e-mail:mostov@itsa.ucsf.edu

\section{Other trafficking books}

Intracellular Cholesterol Trafficking edited by T. Y. Chang and Dale A. Freeman Kluwer Academic Publishers, $£ 105 / \$ 150$

Protein Trafficking in Plant Cells edited by Jurgen Soll Kluwer Academic Publishers, £122/\$175

Adhesion Molecules and Chemokines in Lymphocyte Trafficking edited by Alf Hamann

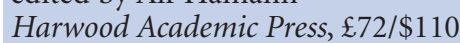

Mechanisms of Intracellular Trafficking and Processing of Proproteins by Y. Peng Loh

CRC Press, $£ 125 / \$ 169$

Receptors: Models for Binding, Trafficking and Signalling

Douglas A. Lauffenburger and Jennifer Linderman

Oxford University Press, $£ 27.50 / \$ 50$

Trafficking of bacterial toxins by C. Saelinger CRC Press, £103/\$153 